\title{
Safe-by-design development of a topical patch for drug delivery
}

\author{
Mafalda. B. Vaz ${ }^{\text {a }}$, Carla Vitorino ${ }^{\circledR a, b, c^{*}}$, João J. S. Sousa ${ }^{a, c}$ \\ ${ }^{a}$ Faculty of Pharmacy, University of Coimbra, Coimbra, Portugal, ${ }^{b}$ Centre for Neurosciences \\ and Cell Biology (CNC), Coimbra, Portugal, ${ }^{c} L A Q V$. REQUIMTE, Group of Pharmaceutical \\ Technology, Faculty of Pharmacy, University of Coimbra, Coimbra, Portugal
}

\begin{abstract}
Few topical products have been developed specifically to treat acute and chronic arthritis and inflammation, using non-steroidal anti-inflammatory drugs (NSAIDs). The lack of dosing accuracy commonly found in locally applied semisolid products for cutaneous use is a critical issue that leads to treatment failure. The aim of the present work is to develop a differentiated and innovative topical patch based on a monolithic hydrogel for ibuprofen skin delivery, in order to provide a safer and accurate way of drug administration along with improved treatment compliance.

Topical patches based on hydroxypropylmethylcellulose (HPMC) were optimized in composition, in terms of enhancer and adhesive, supported on a systematic assessment of in vitro release and permeation behavior and adhesion properties. Several mathematical models were used to scrutinize the release mechanisms from the patches. In vitro release kinetics was shown to be mainly driven by diffusion. However, other mechanisms seemed to be also present, supporting the feasibility of using patches for sustained drug delivery. PEG 200 provided the best permeation rate, with a permeation enhancement ratio of ca. 3 times higher, than the commercial reference. The addition of Eudragit L30D 55 to the formulation led to the best adhesion profile, thus achieving a successful development based on a safe-by-design concept.
\end{abstract}

Keywords: Topical patches. Ibuprofen. Mechanical properties. Permeation studies. Safe-by-design.

\section{INTRODUCTION}

The skin is the largest organ of the human body and a key barrier in drug delivery. It is structured into three main layers: the epidermis, dermis and hypodermis. The epidermis, in particular the outermost layer known as the stratum corneum (SC), represents the main biological barrier in drug delivery. SC is a highly lipophilic membrane described as a brick and mortar structure, with corneocytes representing bricks in a matrix and intercellular lipids, with desmosomes, acting as molecular rivets between the corneocytes and the remaining lipids within a cornified cell. (Benson and

*Correspondence: C. Vitorino. Faculty of Pharmacy. University of Coimbra. Pólo das Ciências da Saúde. Azinhaga de Santa Comba, 3000-548 Coimbra, Portugal. Phone: +351 239488 400. E-mail: csvitorino@ff.uc.pt
Watkinson, 2012). This layer represents a challenging physical barrier, regulating water loss from the body, preventing permeation of microorganisms of the skin surface and restricting passive drug diffusion. (Cox et al., 2008; Elias, 2005 and 2007; Marks, 2004; Menon et al., 2012).

Over time, many compounds have been studied for skin delivery, acting mostly as antibacterials, antifungals, anti-inflammatories and analgesics, with applications typically involving permeation to deeper tissues or targeting the skin surface or appendages. Topical products exhibit advantages regarding the oral formulations, since they can overcome first-pass metabolism and the adverse gastrointestinal tract environment, which is associated to $\mathrm{pH}$ variation, enzymatic activity and also drug interaction with food and drinks (Prausnitz and Langer, 2008). Additionally, they increase the patient compliance due to simpler 
dosage regimens and in case of adverse drug reactions, the treatment can be easily interrupted by removing the formulation from the skin. However, this type of administration has disadvantages, they can stick and stain the clothes, and more importantly can be associated to an inaccurate dose administration, imposing patient safety concerns (Brown et al., 2006).

Ibuprofen (IBU) is one of the most widely used non-steroidal anti-inflammatory drug for pain relief, osteoarthritis, fever reduction and also to minimize acute or chronic pain associated with inflammation (Goldman, 2005; Ong and Seymour, 2007). It is commonly administered by oral route, requiring dosing at least three times daily, deeming from its extensive first-pass metabolism and short elimination half-life (Bushra and Aslam, 2010). However, its repeated oral administration is associated to gastric irritation, contributing to gastric discomfort, nausea and vomiting (Vaile and Davis, 1998) and is also a potential cause of gastrointestinal bleeding that can lead to gastric ulcers (Michels et al., 2012). Moreover, renal failure, apoptosis, heart failure, hyperkalemia, confusion and bronchospasm are also notified as major adverse reactions of IBU (Rainsford, 2015). Topical delivery arises as an alternative route for IBU administration, conventionally encompassing gels, creams and ointments as benchmark semisolid dosage forms. The semisolid drug products have gained their place on the market as a result of the advantages they exhibit in comparison to oral products, such as reduction of doses administrated and avoidance of adverse reactions, due to the ease of formulation removal from the skin. Additionally, these products demonstrate advantages closely related to the patients, since they are non-invasive and not expensive. Recent investigations have reported the use of IBU incorporated into oxidized cellulose (Celebi et al., 2016) or nanostructured lipid carriers (NLC)-based gels (Suto et al., 2016). However, these approaches still lack the requirements a formulation may present to satisfy an application throughout a longer lasting treatment. The application of the safe-bydesign concept consubstantiated by the development of topical patches as drug delivery systems for the skin arises as an appealing strategy, in alignment with the new guidance on "Safety Considerations for Product Design to Minimize Medication Errors". Accordingly, FDA expects the performance of proactive risk assessments along the development of drug products to build safety into drug product throughout its lifecycle and to identify safety characteristics of the product that are considered to be critical. In this way, a proactive risk assessment should start with an evaluation of why and how problems have occurred with similar products and should be conducted before finalizing the physical design features of a drug product. (Bajaj, Kumar, 2016). This concept seeks to minimize potential health risks and hazards by analyzing the full life of the designed product preventing unnecessary setbacks. Because of the limitations of the size of the dermal dose site, in order to be considered safe, the lowest dose should, ideally, cause no systemic effects and minimal or no topical effects (Willard-Mack et al., 2016). Topical drug-in-adhesive patches, by combining both adhesive and drug within a single layer, offer considerable advantages over conventional dosage forms, since there is no need to spread the formulation, which allows an easier, cleaner, and above all, safer application (Bajaj and Kumar 2016; Kathe and Harsha 2017). Apart from the lower safety risk to the patients, the patch administration provides a more accurate dosing, with a undisrupted flow of the drug to the site of action (Prausnitz and Langer, 2008). Indeed, topical patches have gained a positive influence on patient compliance, due to the avoidance of multiple applications per day, whereas for drugs with a short half-life, such as IBU, this type of application can reduce dose frequency up to once a week (Jorge et al., 2011; Jung et al., 2015).

IBU gathers adequate physicochemical properties as drug candidate for skin delivery, since it is a small molecule with a molecular weight of $206 \mathrm{~g} / \mathrm{mol}$, has a $\log \mathrm{P}$ of 3.97 and melting point around the $76{ }^{\circ} \mathrm{C}$. In order to optimize the drug delivery, it is important to understand how the composition of the vehicle influences the partitioning and/or the diffusivity of the drug and consequently the absolute amount delivered (Herkenne and Naik, 2007; Heyneman et al., 2000; Jorge et al., 2011; Moser et al., 2001; Vinod and Sharma, 2014).

The aim of this study is to develop suitable topical patches, supported on a safety-by-design approach. Drug-in-adhesive systems, prepared by solvent casting method (Cherukuri et al., 2017), for incorporation of IBU as model drug for skin delivery will be compared with a commercial reference. Additionally, the systematic assessment of how composition variables, namely, enhancer and adhesive, could impact drug product performance will be explored. A combination of in vitro release, permeation and adhesion studies as critical quality attributes should allow the selection of 
the formulation that best suited target product profile for topical delivery of IBU.

\section{MATERIAL AND METHODS}

\section{Material}

Hydroxypropylmethyl cellulose (HPMC, Methocel ${ }^{\circledR}$ E4M) was a kind gift from Colorcon ${ }^{\circledR}$ (Dartford, England), polyethylene glycol (PEG) 200 was obtained from Fluka, Chemie GmbH (Steinheim, Germany). PEG 300 was acquired from Scharlau (Sentmenat, Barcelona, Spain) and PEG 400 was purchased from Merck (Darmstadt, Germany). IBU was donated from Medinfar (Amadora, Portugal), propylene glycol was obtained from José Manuel Gomes dos Santos, LDA (Odivelas, Portugal) and isopropyl alcohol was purchased from Sigma-Aldrich ${ }^{\circledR}$ (St. Louis, Missouri, USA). Eudragit ${ }^{\circledR}$ L 30 D-55 was provided from Evonik industries (Darmstadt, Germany) and chitosan low molecular weight was a gift from Lusifar Químico Comercial (Lisboa, Portugal).
All other reagents or solvents were from analytical or from high performance liquid chromatography (HPLC) grade.

\section{Patches preparation and characterization}

For the patch preparation, different hydrogels were firstly obtained by solvent casting method using a solution of HPMC $(1.5 \% \mathrm{w} / \mathrm{w})$ prepared under mechanical stirring at $80{ }^{\circ} \mathrm{C}$.

IBU was previously dissolved in a mixture of cosolvents, composed by propylene glycol, isopropyl alcohol and different polyethylene glycols (PEG) 200, 300 or 400 , according to Table I. This solution was added to the former and further mixed under mechanical stirring (400 rpm, EUROSTAR power control-visc, IKA, Staufen, Germany) at a lower temperature $\left(60^{\circ} \mathrm{C}\right)$.

Different adhesive polymers, namely chitosan or Eudragit L30 D-55, were subsequently added to the HPMC solution (Table II).

Finally, patches were obtained by laminating the hydrogels using an Elcometer 3570 • 3580 Film Applicator, adjusted to $3 \mathrm{~mm}$ and dried for 24 hours at $37^{\circ} \mathrm{C}$.

TABLE I - Topical patch composition $\%(\mathrm{w} / \mathrm{w})$ with co-solvent variation

\begin{tabular}{|c|c|c|c|c|c|c|c|c|}
\hline $\begin{array}{c}\text { Formulation } \\
\text { Code }\end{array}$ & Ibuprofen & HPMC & $\begin{array}{c}\text { Propylene } \\
\text { Glycol }\end{array}$ & PEG 200 & PEG 300 & O PEG 400 & $\begin{array}{c}\text { Isopropyl } \\
\text { Alcohol }\end{array}$ & Water \\
\hline $\mathrm{F}_{1}$ & 5 & 1.5 & 20 & - & - & 20 & 20 & 33.5 \\
\hline $\mathrm{F}_{2}$ & 5 & 1.5 & 20 & 20 & - & - & 20 & 33.5 \\
\hline $\mathrm{F}_{3}$ & 5 & 1.5 & 20 & - & 20 & - & 20 & 33.5 \\
\hline
\end{tabular}

TABLE II - Topical patch composition $\%(w / w)$ with addition of adhesive polymer

\begin{tabular}{ccccccccc}
\hline $\begin{array}{c}\text { Formulation } \\
\text { Code }\end{array}$ & Ibuprofen & HPMC & $\begin{array}{c}\text { Propylene } \\
\text { Glycol }\end{array}$ & $\begin{array}{c}\text { PEG } \\
\mathbf{2 0 0}\end{array}$ & $\begin{array}{c}\text { Isopropyl } \\
\text { Alcohol }\end{array}$ & Water & Chitosan & Eudragit \\
\hline $\mathrm{F}_{4}$ & 5 & 1.5 & 20 & 20 & 20 & 25 & 7,5 & - \\
$\mathrm{F}_{5}$ & 5 & 1.5 & 20 & 20 & 20 & 32 & - & 1.5 \\
\hline
\end{tabular}




\section{Adhesion properties}

The evaluation of the in vitro adhesion properties of the patches were determined by tack adhesion, tensile strength and elongation to break tests, using a Texture Analyzer TA.XT Plus (Stable Micro Systems Ltd., Surrey, UK).

Tack is described as the maximum force required to break a bond formed under low contact pressure between the adhesive layer of the patch and a stainless steel probe. The tack adhesion set up test consists of one stainless steel ball probe $(\mathrm{P} / 1 \mathrm{~S})$, that ensures contact consistency with the adhesive, and a strip of doublesided tape, sticked to the Heavy Duty plate, where the sample is applied (Benson, Watkinson, 2012).

To evaluate the adhesion and the resistance to separation between the probe and the sample, the probe is brought into contact with the patch and the force is recorded while the probe is being pulled away. Six replicates were carried out with data collection and calculation being performed using the Texture Exponent 3.0.5.0 software package of the instrument. The results are represented as mean \pm standard deviation (SD).

\section{Mechanical properties}

To perform tensile strength (TS) and elongation to break (EB \%), the samples were cut into strips with 73 $\mathrm{mm}$ length and $37 \mathrm{~mm}$ width and the TA.XT Plus Texture analyzer was equipped with a tension grip system.

The TS is calculated by dividing the maximum breaking force $(\mathrm{N})$ by the cross sectional area $\left(\mathrm{mm}^{2}\right)$ of each patch (Caner et al., 1998). Six replicates were taken on each patch considering different places. Mean values and standard deviations were calculated for each patch TS.

In turn, EB (\%) is the ratio between the final length at the point of rupture and the initial length of the sample and is expressed in percentage (Padula et al., 2003).

\section{In vitro release studies}

The release studies were performed with Franz cells (PermeGear, Inc., PA, USA) with a diffusion area of $0.636 \mathrm{~cm}^{2}$ and a receptor compartment of $5 \mathrm{~mL}$ filled in with PBS $(\mathrm{pH}=7.4)$. The receptor medium was stirred at $600 \mathrm{rpm}$ and thermo-regulated at $37 \pm 0.5{ }^{\circ} \mathrm{C}$ (ensuring $32{ }^{\circ} \mathrm{C}$ at the membrane surface to mimic skin conditions) with a water pump, which circulated water through each chamber jacket. This medium ensured the maintenance of sink conditions. A dialysis cellulose membrane (MWCO 12,000, avg. flat width $33 \mathrm{~mm}$, D9652, Sigma-Aldrich), used as artificial membrane, was placed between both compartments (Vitorino et al., 2013).

The patch samples and the control reference were applied to the donor compartment and occluded with Parafilm ${ }^{\circledR}$ to prevent evaporation. Ozonol gel $(50 \mathrm{mg} / \mathrm{g})$ is specially formulated for pain and inflammation relief associated with mild to moderate muscle pain. Since contains IBU, propylene glycol and ethanol, was used as commercial reference for comparison purposes. The release studies were carried out for $24 \mathrm{~h}$ and at 8 predetermined time points, aliquots of $300 \mu \mathrm{L}$ of the receptor compartment were collected and replaced with fresh medium.

All drugs were determined using the HPLC method described below.

\section{In vitro permeation studies}

The in vitro permeation studies were performed in the same Franz cells conditions of the in vitro release studies, but instead of the dialysis cellulose membrane, a newborn pig epidermis was used as skin model, with the stratum corneum side facing up (Iqbal et al., 2017).

The newborn pig, provided by a local slaughterhouse, was sectioned and the subcutaneous fat removed. The heat separation technique was used to separate the epidermis from the underlying dermis. This technique consists in the immersion of the entire skin in hot water, around $60^{\circ} \mathrm{C}$, for one minute and gently, with the help of forceps, pulled the epidermal layer. This layer separation is more appropriate for permeants that are poorly water soluble (Benson, Watkinson, 2012).

Epidermis was further cut in pieces of $2.5 \times 2.5$ $\mathrm{cm}^{2}$, wrapped with aluminum foil and stored at $-20{ }^{\circ} \mathrm{C}$ until being used.

The barrier function of the skin was monitored by measuring the transepidermal water loss (TEWL) and only the pieces that retrieved values below $20 \mathrm{~g} / \mathrm{m}^{2} \mathrm{~h}$ were used.

\section{Calculations}

The cumulative amount of ibuprofen diffused per unit area of the excised skin (Qn) is 


$$
Q n=\left(C_{n} \times V_{0}+\sum_{i=1}^{n-1} \mathrm{Cix} V i\right) / A
$$

expressed in $\mu \mathrm{g} / \mathrm{cm}^{2}$, was plotted as a function of time $(t, h)$. In this equation $C_{n}$ corresponds to the drug concentration of the receptor medium at each sampling time, $\mathrm{C}_{\mathrm{i}}$ is the drug concentration of the sample, $\mathrm{A}$ is the effective diffusion area, $\mathrm{V}_{0}$ stand for the volume of the receptor compartment and $\mathrm{V}_{\mathrm{i}}$ for the volume of the sample. The total quantities of the drugs obtained after 24 h (Q24) were used for comparison among formulations. The slope of the linear region on the representation of the amount of drug permeated by unit area versus time was used to calculate the flux at steady state (Vitorino et al., 2013).

According to Fick's first law of diffusion, the flux $\left(\mu \mathrm{g} / \mathrm{cm}^{2} / \mathrm{h}\right)$ can be expressed by

$$
J s s=\frac{\mathrm{C}_{0} \mathrm{KD}}{\mathrm{L}}=\mathrm{C}_{0} \mathrm{Kp}
$$

where represents the drug concentration in the donor compartment, $\mathrm{K}$ the partition coefficient of drug between membrane and vehicle, D stands for the diffusion coefficient and $\mathrm{L}$ for the thickness of the membrane. The permeability coefficient is represented by $\mathrm{K}_{\mathrm{p}}$. The lag time of permeation, $\mathrm{t}_{\mathrm{lag}}$, is a parameter related to the time required to achieve the steady-state flux of a drug through the skin was also taken into consideration. It was determined from the extrapolation of the linear portion of the plot to the $\mathrm{x}$-axis [30].

In the case of the release studies, only the cumulative percentage of drug was taken into consideration and represented, following an approach similar to that described above for the permeation assays.

The enhancement ratio (ER) for flux was calculated as the ratio between the flux/ permeability coefficient/ Q24 for treated skin with enhancer(s)/adhesive polymer(s) and flux/ permeability/Q24 coefficient for Ozonol.

\section{HPLC determination of IBU}

The quantification of IBU was performed using a reversed phase high-performance liquid chromatography (HPLC) method, by a Shimadzu LC-2010 CHT apparatus equipped with a quaternary pump, an auto-sampler unit, a CTO-10AS oven and a SPD-M2OA detector. The column used for the analysis was a RP18 (4.6 mm x 125 $\mathrm{mm}$ ) Lichrospher ${ }^{\circledR} 100$ analytical column (Merck KGaA, Germany), equipped with a guard column (Purospher ${ }^{\circledR}$ STAR RP-18 endcapped, $5 \mu \mathrm{m}$ ).

Mobile phase consisted of a 60:40 (v/v) mixture of acetonitrile:water adjusted to $\mathrm{pH} 2.7$ with orthophosphoric acid with a flow rate of $1.0 \mathrm{~mL} / \mathrm{min}$, an injection volume of $20 \mu \mathrm{L}$ and detection at $221 \mathrm{~nm}$ with the temperature set at $30{ }^{\circ} \mathrm{C}$.

A run of 6 minutes was stablished and the ibuprofen eluted at 2.4 minutes.

\section{Determination of $\mathrm{pH}$}

As a quality control parameter, the $\mathrm{pH}$ of the relevant formulations was measured, using a digital pH meter Consort C3010 (Dias de Sousa, Portugal), previously calibrated through buffer solutions with $\mathrm{pH}$ of 4.00, 7.00 and 10.01 .

\section{IBU assay in hydrogels and patches}

A pre-defined amount ( $5 \mathrm{~g}$ ) of hydrogel formulation was dissolved in $5 \mathrm{~mL}$ of mobile phase solution and kept under agitation overnight. The solutions were filtered $(0.45 \mu \mathrm{m})$ and appropriate dilutions were done in order to measure the drug content using the HPLC method previously described in section 2.2.5. IBU was also extracted from Ozonol, the commercial reference, for comparison purpose, using the same procedure.

For quantification of IBU in patches, a pre-defined area $\left(1.44 \mathrm{~cm}^{2}\right)$ of the patch was cut, suitably diluted in mobile phase, and kept under orbital agitation until complete dispersion. The samples were filtered $(0.45$ $\mu \mathrm{m})$ and IBU quantified by HPLC.

\section{Statistical analysis}

The significance of differences was evaluated using both the F test and student's t test at the significance level of 0.05 . This analysis was performed using Microsoft Excel (Microsoft Corp., Redmond, WA, USA).

\section{RESULTS AND DISCUSSION}

The design of a quality topical patch product and its manufacturing process are described in the following 
sections. A systematic development approach based on the assessment of release, permeation behavior and adhesion properties, as critical quality attributes (CQAs), was taken into consideration to consistently deliver the intended performance of the product.

\section{Process variables}

Temperature, time of agitation and patch thickness were firstly identified as critical process parameters (CPP) within the process of manufacturing. This analysis and further optimization track assume crucial importance, since these are parameters whose variability impacts on CQAs, such as viscosity, content uniformity, and release profile, and, therefore, should be monitored or controlled to ensure the process leads to the desired quality product.

Although the patch preparation began with a solution of HPMC $(1.5 \% \mathrm{w} / \mathrm{w})$ prepared under mechanical stirring at $80^{\circ} \mathrm{C}$, lower temperatures were previously considered, since this parameter is closely related to the polymer concentration. In addition, it has been proved (Sarkar, 1979; Silva et al., 2008b) that when the temperature rises, there is a smoothly decrease of viscosity until the gelation temperature is reached. After this temperature, ca. $75{ }^{\circ} \mathrm{C}$ in the case of HPMC, the viscosity increases keenly. This characteristic temperature is concentration dependent, tending to decrease at concentrations higher than $2 \% \mathrm{w} / \mathrm{w}$ (Silva et al., 2008b). For this reason, a HPMC concentration of $1.5 \% \mathrm{w} / \mathrm{w}$ was chosen. Note that ensuring an appropriate viscosity is highly desirable, since it strongly influences the intrinsic hydrogel rheological and film-forming properties, consequently impacting formulation homogeneity and drug release (Simoes et al., 2018).

Topical patches adjusted to a thickness of $3 \mathrm{~mm}$ and, exhibiting a thin, smooth and transparent appearance enabled to ensure the product performance, bearing in mind that thicker patches would hamper the patient acceptability and could condition the safety-by-design principle. The patch composition was further optimized, so as to achieve the best adhesive properties and promote an improvement of drug in vitro release and permeation.

\section{Formulation variables: enhancer molecular weight}

Formulation optimization relied on the study of the influence of enhancer and adhesive in patch composition by means of performance testing in terms of release, permeation and adhesion/mechanical properties CQAs. Again, these are quality related issues that might directly or indirectly dictate the in vivo release characteristics of a topical patch.

\section{In vitro release studies}

The evaluation of topical drug products through pharmacopeial and nonpharmacopeial tests ensure their efficacy and safety characteristics. According to the Pharmacopeial Forum product quality-test recommendations, drug product in process, release, and stability tests should be as complete as possible (Chang et al., 2015).

The release behavior of IBU from three different patches containing PEG 200, PEG 300 or PEG 400, respectively, was assessed using the same conditions previously described.

It can be observed (Figure 1) that patches $\mathrm{F}_{2}$ and $\mathrm{F}_{3}$, with PEG 200 and 300 respectively, showed very similar profiles, reaching both approximately $50 \%$ IBU release after 24 hours, while for $\mathrm{F}_{1}$ (PEG 400) lower values (around 45\%) were obtained. Such a behavior could be attributed to the decreasing trend in the molecular weight (MW) of PEGs employed, since a lower MW is associated to a shorter chain length (Bolourchian et al., 2013). If the polymer chain is shorter, the entrapment of the drug is compromised, therefore resulting in a higher release profile of IBU.

However, without showing any significant differences $(p>0.05)$, it can be observed that all the profiles still indicate smaller release values than the commercial reference.

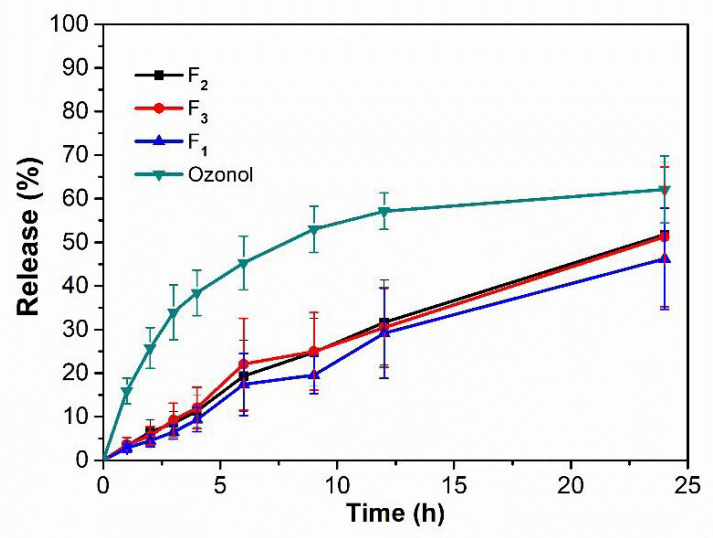

FIGURE 1 - In vitro release profiles of IBU from patch formulations. The results are expressed as mean \pm SEM $(\mathrm{n}=6) .{ }^{*} \mathrm{p}<0.05$ vs. Ozonol. 


\section{In vitro permeation studies}

Chemical penetration enhancers (CPE) are compounds that may potentially change the solubility/ partitioning behaviour of the drug into the SC and/or its diffusion properties. According to the lipid-proteinpartitioning (LPP) theory, the mechanisms of drug penetration enhancement relies on interactions with the intercellular lipids, the intracellular keratin and, finally, the penetration of high amounts of enhancers or co-solvents into the SC, thus improving the dissolution capacity of the barrier for drugs and/or enhancers (Alexander et al., 2012).

Permeation studies were performed to assess the impact of the PEG molecular weight on drug permeation. The PEGs and propylene glycol, both CPP, are usually incorporated in topical and transdermal patches, in order to modify the barrier properties of the $\mathrm{SC}$ and enhance the drug penetration and absorption. These enhancers improve the diffusivity and solubility of drugs through the skin that would reversibly reduce the barrier resistance of the SC allowing the drug to penetrate to the viable tissues (Aukunuru et al., 2007; Valenta et al., 2000).

The results obtained are displayed in Figure 2 and the permeation parameters calculated from the obtained profiles are presented in Table III.

As it can been seen in the profiles obtained, the incorporation of PEG is important to achieve a controlled delivery, which allows a less frequent dosing
(Frederiksen et al., 2016), without compromising the amount of IBU permeated in the end of the study.Topical patch profiles evidence different permeation behviour, with $F_{2}$ (PEG 200) and $F_{3}$ (PEG 300) formulations leading to higher values of the IBU amount permeated than $F_{1}$ (PEG 400). These results reinforce the impact of lower MW PEG in the retention of the drug within the polymeric matrix, therefore resulting in a higher release and permeation of IBU. Interestingly, all formulations exhibited better permeation rate than Ozonol, with statistically significant differences at 24 hours, $(p<0.05)$. Such a behavior can be ascribed to the presence of enhancer along with the close contact and consequent occlusion provided by the patch in comparison to gel formulation. Note that, the dosage form can influence the amount of drug permeated, since the patch provides a continuous flow of the drug to the site of action.

These findings are consistent with data from other works (Luo et al., 2016), with $\mathrm{F}_{3}$ reaching ca. $35 \%$ and $\mathrm{F}_{2} 40 \%$ of IBU permeation. Therefore PEG 200 based formulation was selected to proceed the studies, since it yielded the highest permeation percentage, flux and permeability enhancement ratios.

Lag time was not taken as a major parameter for the enhancer screening, with a lagging behavior absent in most profiles. IBU was mainly detected in the receptor compartment at $24 \mathrm{~h}$ post skin application, but started being released from the formulation in the first hour of study.

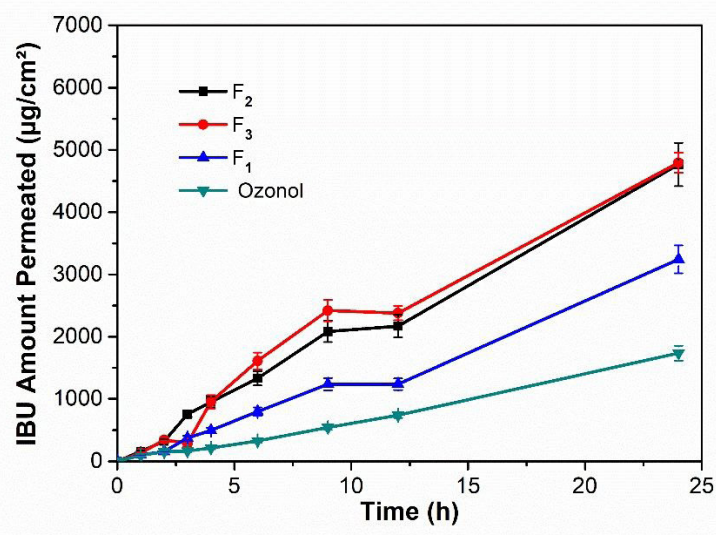

FIGURE 2 - In vitro permeation profiles of IBU from patch formulations. The results are expressed as mean $\pm \operatorname{SEM}(\mathrm{n}=6) * \mathrm{p}<$ 0.05 vs. Ozonol. 
TABLE III - Formulations and respective permeation parameters, according to in vitro permeation studies in epidermis. The $\mathrm{J}_{\mathrm{ss}}$ represents the flux at steady-state; $\mathrm{K}_{\mathrm{p}}$ the Permeability coefficient; Q24 the Cumulative amount of IBU permeated after $24 \mathrm{~h}$. Data are expressed as mean $\pm \operatorname{SEM}(\stackrel{\mathrm{p}}{\mathrm{n}}=6)$

\begin{tabular}{ccccccc}
\hline Formulations & $\begin{array}{c}\mathbf{J}_{\text {ss }} \\
\boldsymbol{\mu g} /\left(\mathbf{c m}^{2} \mathbf{h r}\right)\end{array}$ & $\mathbf{E R ~ J ~}_{\mathbf{s s}}$ & $\begin{array}{c}\mathbf{K}_{\mathbf{p}} \\
(\mathbf{c m} / \mathbf{h})\end{array}$ & $\begin{array}{c}\mathbf{E R} \\
\mathbf{K p}\end{array}$ & $\begin{array}{c}\mathbf{Q 2 4} \\
\left(\boldsymbol{\mu g} / \mathbf{c m}^{2}\right)\end{array}$ & $\begin{array}{c}\mathbf{E R} \\
\mathbf{Q} 24\end{array}$ \\
\hline $\mathbf{F}_{\mathbf{1}}$ & $148 \pm 36$ & 1.21 & $0.011 \pm 0.003$ & 1.10 & $3242 \pm 599$ & 1.87 \\
$\mathbf{F}_{\mathbf{2}}$ & $186 \pm 2$ & 1.52 & $0.015 \pm 0.003$ & 1.70 & $4866 \pm 930$ & 2.79 \\
$\mathbf{F}_{\mathbf{3}}$ & $186 \pm 22$ & 1.52 & $0.017 \pm 0.004$ & 1.50 & $5121 \pm 463$ & 2.95 \\
Ozonol & $122 \pm 13$ & 1.00 & $0.010 \pm 0.001$ & 1.00 & $1736 \pm 147$ & 1.00 \\
\hline
\end{tabular}

\section{Formulation variables: adhesive properties}

In order to achieve the purpose of the development of a topical patch, one of the critical issues is the adhesive properties of the patch.

The lack of adhesion can make the patch unusable and it can be related to environmental conditions, such as heat or cold and due to poor quality of the patch itself when the edges result in curling up or patch not sticking after $24 \mathrm{~h}$ (Cilurzo et al., 2012). This is a critical aspect, being part of the risk analysis of topical patch dosage forms. An adequate adhesion to skin is of primary importance for effective percutaneous absorption. When patches involuntarily detach, the therapy is disrupted, thus imposing a risk of exposure to third parties, besides the financial burden on patients resulting from their frequent replacement (Kakhi et al., 2007).

A screening was conducted for the selection of adhesive polymer and chitosan and Eudragit L30 D-55 were chosen, since both have been commonly incorporated in transdermal patches.

Chitosan is a natural polycationic polysaccharide, which has favorable features, such as biocompatibility, non-toxicity to living tissues and antibacterial and antifungal properties (Dash et al., 2011). Additionally, chitosan can improve the penetration of drug compounds by opening the tight junctions as a result of the chitosan ability to enhance the paracellular permeability of mucosal membranes (Can et al., 2013).

On the other hand, Eudragit L30 D-55, which is a low viscosity aqueous dispersion of anionic polymers with methacrylic acid as a functional group, has been in the last 15 years reportedly used with permeation enhancers for dermal and transdermal patches. In this context, it can be mentioned the study carried out by Nesseem and co-workers (Nesseem et al., 2011), who used Eudragit L30 D-55 along with permeation enhancers, like PEG 200 and 400, and propylene glycol, for the design of transdermal patches of tenoxicam.

\section{In vitro release studies}

To assess how the incorporation of an adhesive would influence the IBU release from the patches, studies under the same conditions were performed as substituir por previously described, and the achieved results are presented in Figure 3.

The chitosan in a ratio of 1:5 of the HPMC (HPMC: Chitosan), and the Eudragit L30 D-55 in same proportion as HPMC, were added to the base composition, as it can be seen in Table II, and their influence on adhesion was investigated.

The patches prepared are thin, smooth and transparent to slightly yellowish (in case of chitosan 
based patches) in appearance. Note that, these are important characteristics to patient acceptability.

The $\mathrm{pH}$ of formulations $\mathrm{F}_{1}-\mathrm{F}_{5}$ was measured using a digital pH meter Consort C3010 (Dias de Sousa, Portugal), previously calibrated. The values of $\mathrm{pH}$ were practically identical ranging from $4.5-4.7$, being appropriate to topical use, since they are compatible with physiological skin conditions (Schmid-Wendtner and Korting, 2006).

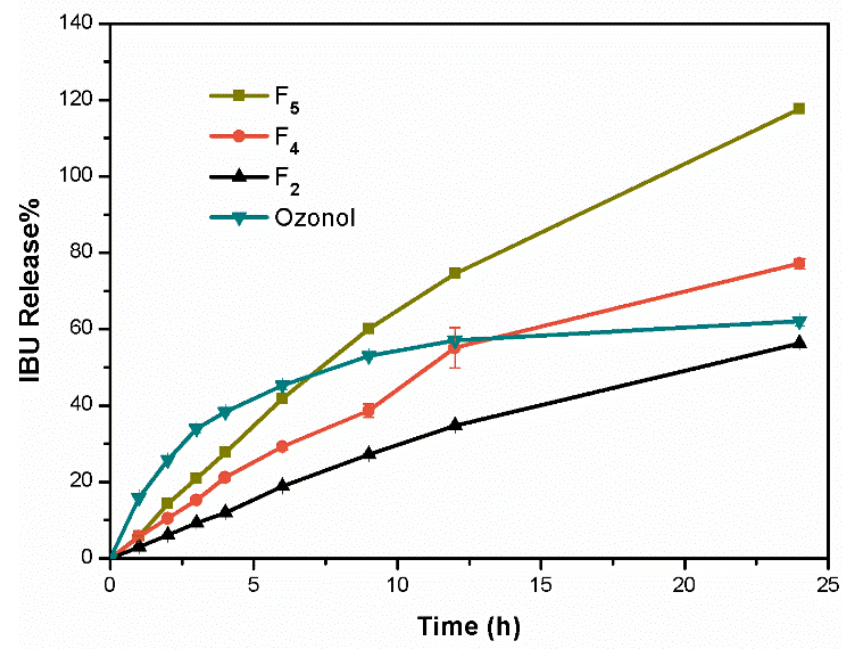

FIGURE 3 - In vitro release profiles of patch formulations with different adhesives. The results are expressed as mean \pm SEM $(n=6)$.

From Figure 3, it can be observed that the inclusion of both chitosan and Eudragit enhance IBU release, even exceeding the amount obtained with Ozonol formulation. Moreover, after $24 \mathrm{~h}$, all the formulations led to higher extent of IBU release than $\mathrm{F}_{2}$, the comparative patch without adhesive.

The formulation with chitosan promoted the highest IBU release percentage, being statistically different from all the formulations tested. This might be explained by an increased area of contact between the patch and the dialysis membrane, deeming from the mucoadhesive properties of this polymer, since it can improve the penetration of drug compounds, as previously mentioned.

The profiles obtained were fitted using different mathematical models, in order to explain the mechanism of drug release, see Table IV (Heyneman et al., 2000) The quality of fitting was assessed according to the value of coefficient of determination, the $\mathrm{R}^{2}$, which should be as closest as possible or even one. According to this principle, Weibull, following by Korsmeyer-Peppas and the first order were the best-fitted models. The fitting for Weibull allows characterizing dissolution profiles by the shape of the curve through $\mathrm{c}_{3}$ parameter, which can be considered exponential, if $\mathrm{c}_{3}=1$; sigmoidal, if $\mathrm{c}_{3}>1$ or parabolic if the $c_{3}<1$ (Costa and Sousa Lobo, 2001). According to the achieved results, formulation $\mathrm{F}_{5}$ displayed a sigmoidal shape, $\mathrm{F}_{4}$, a parabolic shape, while $\mathrm{F}_{2}$ and Ozonol resembled to an exponential trend.

The prediction of the drug transport mechanism is made through the results obtained in KorsmeyerPeppas with the $c_{2}$ values. If they are under, but close to 0.5 , they correspond to a Fickian diffusion process. On the contrary, if they are above, in the 0.5 and 1.0 range, they correspond to an anomalous (non-Fickian) transport, probably associated to polymer matrix relaxation. When the $c_{2}$ value is 1.0 a zero-order model is applied (Idson, 1975). Bearing in mind this analysis, results obtained were all above 0.5 but under 1.0 , corresponding to a non-Fickian transport, where diffusion is attached with other mechanisms, with the exception of Ozonol, which, due to a value of 1.170 is associated to super case II transport. However, this semi-empirical model has a disadvantage, since it just considers the first $60 \%$ of the release.

The last fitting presenting a good $\mathrm{R}^{2}$ values was the first-order model, where the $\mathrm{c}_{1}$ parameter corresponds to the asymptotic value predicted for each profile. This parameter was higher for the $\mathrm{F}_{4}$ in comparison with all the formulations and the corresponding release rates were also larger, as extracted from the $c_{2}$ values, which are in agreement with the results obtained. 
TABLE IV - Regression parameters resulting from the application of the different mathematical models to the experimental release data

\begin{tabular}{|c|c|c|c|c|c|}
\hline Function & Formulation & $\mathbf{c}_{1}$ & $\mathbf{c}_{2}$ & $\mathbf{c}_{3}$ & $\mathbf{R}^{2}$ \\
\hline Zero Order & Ozonol & $0.763 \pm 2.651 \times 10^{-2}$ & - & - & 0.9884 \\
\hline \multirow[t]{3}{*}{$c_{1 .} \mathrm{T}$} & $\mathrm{F}_{2}$ & $1.858 \pm 5.351 \times 10^{-2}$ & - & - & 0.9892 \\
\hline & $\mathrm{F}_{4}$ & $1.577 \pm 1.449 \times 10^{-1}$ & - & - & 0.9500 \\
\hline & $\mathrm{F}_{5}$ & $2.124 \pm 1.160 \times 10^{-1}$ & - & - & 0.9621 \\
\hline First Order & Ozonol & $3.640 \times 10^{3} \pm 1.991 \times 10^{5}$ & $2.089 \times 10^{-4} \pm 1.145 \times 10^{-2}$ & - & 0.9883 \\
\hline \multirow[t]{3}{*}{$c_{1}\left(1-\exp \left(-c_{2} t\right)\right)$} & $\mathrm{F}_{2}$ & $2.190 \times 10^{2} \pm 1.918 \times 10^{2}$ & $9.246 \times 10^{-3} \pm 8.831 \times 10^{-3}$ & - & 0.9901 \\
\hline & $\mathrm{F}_{4}$ & $3.802 \times 10^{1} \pm 5.113$ & $7.547 \times 10^{-2} \pm 1.748 \times 10^{-2}$ & - & 0.9757 \\
\hline & $\mathrm{F}_{5}$ & $1.666 \times 1^{-2} \pm 1.712 \times 10^{2}$ & $1.459 \times 10^{-2} \pm 1.715 \times 10^{-2}$ & - & 0.9710 \\
\hline Higuchi & Ozonol & $2.660 \pm 4.206 \times 10^{-1}$ & - & - & 0.8415 \\
\hline \multirow[t]{3}{*}{$c_{1 .} t^{0.5}$} & $\mathrm{~F}_{2}$ & $6.703 \pm 7.893 \times 10^{-1}$ & - & - & 0.9099 \\
\hline & $\mathrm{F}_{4}$ & $6.092 \pm 2.222 \times 10^{-1}$ & - & - & 0.9837 \\
\hline & $\mathrm{F}_{5}$ & $7.599 \pm 1.043$ & - & - & 0.8874 \\
\hline Weibull & Ozonol & $1.703 \times 10^{3} \pm 9.023 \times 10^{4}$ & $9.156 \times 10^{-4} \pm 4.217 \times 10^{-2}$ & $1.172 \pm 1.914 \times 10^{-1}$ & 0.9973 \\
\hline \multirow[t]{3}{*}{$c_{1}\left(1-\exp \left(-c_{2} t^{t^{3}}\right)\right)$} & $\mathrm{F}_{2}$ & $5.313 \times 10^{2} \pm 5.544 \times 10^{3}$ & $3.244 \times 10^{-3} \pm 3.873 \times 10^{-2}$ & $9.62 \times 10^{-1} \pm 2.46 \times 10^{-1}$ & 0.9904 \\
\hline & $\mathrm{F}_{4}$ & $1.198 \times 10^{3} \pm 3.049 \times 10^{4}$ & $1.270 \times 10^{-4} \pm 5.428 \times 10^{-3}$ & $6.20 \times 10^{-1} \pm 1.31 \times 10^{-1}$ & 0.9945 \\
\hline & $\mathrm{F}_{5}$ & $4.907 \times 10^{1} \pm 2.144$ & $8.479 \times 10^{-2} \pm 5.314 \times 10^{-3}$ & $1.904 \pm 1.855 \times 10^{-1}$ & 0.9962 \\
\hline Korsmeyer-Peppas & Ozonol & $0.469 \pm 5.614 \times 10^{-2}$ & $1.170 \pm 4.043 \times 10^{-2}$ & - & 0.9973 \\
\hline \multirow[t]{3}{*}{$\mathrm{c}_{1} \mathrm{t}^{\mathrm{c}^{2}}$} & $\mathrm{~F}_{2}$ & $2.195 \pm 3.36418 \times 10^{-1}$ & $9.412 \times 10^{-1} \pm 5.352 \times 10^{-2}$ & - & 0.9905 \\
\hline & $\mathrm{F}_{4}$ & $4.589 \pm-3.051 \times 10^{-1}$ & $6.160 \times 10^{-1} \pm 2.516 \times 10^{-2}$ & - & 0.9946 \\
\hline & $\mathrm{F}_{5}$ & $2.387 \pm 7.672 \times 10^{-1}$ & $9.589 \times 10^{-1} \pm 1.119 \times 10^{-1}$ & - & 0.9645 \\
\hline
\end{tabular}




\section{Mechanical and adhesion properties}

Using the TA.TX Plus Texture Analyzer and the tensile grips, it is possible to evaluate the mechanical properties used to describe the patches in terms of their resistance to abrasion, with tensile strength (TS), and flexibility, measure with the elongation to break test. These properties are determinant, since they can affect the patient compliance and can influence the patient daily routine. The results are expressed in Table V along with the adhesive properties.

In vitro adhesion tests are used to characterize the adhesion of patches, being considered critical quality attributes. These tack tests assess the maximum force required to break a bond formed under pressure between the patch and the steel probe. The adhesiveness was measured by "Transdermal Adhesive Tape", through tack test parameters, such as adhesiveness, energy of adhesion and distance to separation.

TABLE V - Mechanical and adhesion properties of different patches with different adhesives. Results are expressed in Mean \pm SEM with $\mathrm{n}=6$

\begin{tabular}{cccccc}
\hline Formulations & $\begin{array}{c}\text { Elongation } \\
\text { to break } \\
(\mathbf{\%})\end{array}$ & $\begin{array}{c}\text { Tensile } \\
\text { Strengh } \\
\mathbf{( N / \mathbf { m m } ^ { 2 } )}\end{array}$ & $\begin{array}{c}\text { Adhesiveness } \\
\mathbf{( k g )}\end{array}$ & $\begin{array}{c}\text { Distance to } \\
\text { Separation } \\
(\mathbf{m m})\end{array}$ & $\begin{array}{c}\text { Energy of } \\
\text { Adhesion } \\
\text { (kg.sec) }\end{array}$ \\
\hline $\mathrm{F}_{4}$ & 83.64 & 0.004 & $0.008 \pm 0.001$ & $0.20 \pm 0.04$ & $1.40 \pm 0.24$ \\
$\mathrm{~F}_{5}$ & 54.46 & 0.005 & $0.006 \pm 0.001$ & $0.02 \pm 0.01$ & $0.32 \pm 0.01$ \\
$\mathrm{~F}_{2}$ & 66.79 & 0.003 & $0.008 \pm 0.001$ & $0.06 \pm 0.03$ & $0.60 \pm 0.08$ \\
\hline
\end{tabular}

It is important that this type of dosage forms possess some characteristics, such as enough flexibility to follow the movements of the skin and capacity to resist to the mechanical abrasion caused by clothes. Thus, a patch should be hard, which is a reflex of a high TS, and should be tough, demonstrating a high percentage of EB (Silva et al., 2008a).

A higher value of elongation to break dictates higher flexibility properties of the patch. Taking this into consideration, it is observed that the formulation $\mathrm{F}_{4}$ has more flexibility than $\mathrm{F}_{5}$ and $\mathrm{F}_{2}$, the corresponding one without adhesive. The Eudragit L30 D55 based patch exhibits even lower values than the $\mathrm{F}_{2}$ although presenting an improved TS.

The results presented for tack adhesion show that the adhesiveness do not suffer significant variation among formulations, but in terms of energy of adhesion, for $\mathrm{F}_{4}$, a stronger force is required to separate the probe from the adhesive patch. Associated with a high value of energy of adhesion is a longer distance of separation, which is in agreement to results obtained.

Despite of high residue shown by the distance to separation parameter, the $\mathrm{F}_{4}$ was chosen as the best adhesive tested formulation, since it presented a higher energy of adhesion and also higher results of elongation to break.

\section{In vitro permeation studies}

To assess the impact of the adhesive polymer on IBU amount permeated from patches, another in vitro skin permeation study was performed, now taking the formulation without adhesive $\left(\mathrm{F}_{2}\right)$ and the commercial one (Ozonol) as references (Figure 4). 


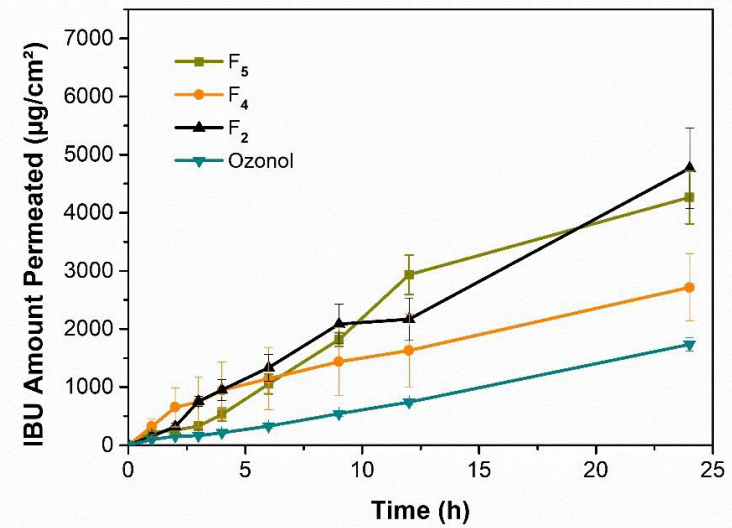

FIGURE 4 - In vitro permeation profiles of patches formulations with the best adhesives. The results are expressed as mean \pm $\operatorname{SEM}(n=6)$.

Unexpectedly, the results achieved in the permeation studies reversed the release profiles obtained in Figure 3. The $\mathrm{F}_{2}$, which promoted the lowest percentage of release, corresponds now to the higher permeation rate, followed by Eudragit formulation $\left(\mathrm{F}_{5}\right)$ and finally chitosan based formulation. This trend could be ascribed to the chitosan patch formation properties, already established for wound healing (Cox et al., 2008; Silva et al., 2008a). Despite the bioadhesive properties of chitosan pointing to an increase in drug penetration, its patch formation ability suggests that the drug is retained at the surface, not allowing it to permeate to deeper skin layers.
On the other hand, the combination of HPMC with Eudragit has already been described to promote increased drug permeation rates (Table VI). As reported in the study carried out by Irfani et al (2011), the permeability coefficients and flux $\left(\mathrm{J}_{s s}\right)$ follow the same trend, exhibiting a better permeation outcome for $\mathrm{F}_{5}$. Moreover, comparing the flux of formulations, with and without adhesive, with other studies (Tombs et al., 2017) involving IBU and propylene glycol as enhancer, all developed formulations showed extensively higher values, with a 30 fold higher flux for the Eudragit formulation.

The $\mathrm{pH}$ obtained for the formulations was compatible with physiological skin conditions, ranging from 4,5 to 4.7 .

Different theoretical mathematical models were used to estimate IBU values of permeability coefficient, based on physicochemical properties of the drug, such as molecular weight and coefficient partition (log P) (Menon et al., 2012), as it can be seen from Table VII.

The predicted permeability coefficients varied from 0.009 to 0.026 , which are within the experimental range of $\mathrm{Kp}$ values. These variations can be ascribed to factors that impact the drug permeability, such as the vehicle type and temperature.

Overall, it highlights that the strategy employed for the pharmaceutical development of the patch, supported on both enhancement and adhesive properties as critical quality attributes led to a better performance for skin IBU delivery.

TABLE VI - In vitro permeation parameters for patches containing adhesive and Ozonol. The results are expressed as mean \pm $\operatorname{SEM}(n=6)$

\begin{tabular}{ccccccc}
\hline Formulations & $\begin{array}{c}\mathbf{J}_{\text {ss }} \\
\boldsymbol{\mu g} /\left(\mathbf{c m}^{2} \mathbf{h r}\right)\end{array}$ & $\mathbf{E R ~ \mathbf { J } _ { \text { ss } }}$ & $\begin{array}{c}\mathbf{K}_{\mathbf{p}} \\
(\mathbf{c m} / \mathbf{h})\end{array}$ & $\begin{array}{c}\mathbf{E R} \\
\mathbf{K p}\end{array}$ & $\begin{array}{c}\mathbf{Q 2 4} \\
\left(\boldsymbol{\mu g} / \mathbf{c m}^{2}\right)\end{array}$ & $\begin{array}{c}\mathbf{E R} \\
\mathbf{Q 2 4}\end{array}$ \\
\hline $\mathrm{F}_{4}$ & $69 \pm 25$ & 0.57 & $0.009 \pm 0.003$ & 0.90 & $2714 \pm 707$ & 1.56 \\
$\mathrm{~F}_{5}$ & $133 \pm 45$ & 1.08 & $0.010 \pm 0.003$ & 1.00 & $4266 \pm 559$ & 2.46 \\
$\mathrm{~F}_{2}$ & $186 \pm 22$ & 1.52 & $0.015 \pm 0.003$ & 1.50 & $4766 \pm 930$ & 2.75 \\
Ozonol & $122 \pm 13$ & 1.00 & $0.010 \pm 0.001$ & 1.00 & $1736 \pm 147$ & 1.00 \\
\hline
\end{tabular}


TABLE VII - Estimated Ibuprofen values of $\mathrm{K}_{\mathrm{p}}(\mathrm{cm} / \mathrm{h})$, from different permeation correlation equations (Hadgraft and Guy, 2003)

\begin{tabular}{lcc}
\hline Equations & Permeation Correlation & $\mathbf{K}_{\mathbf{p}}(\mathbf{c m} / \mathbf{h})$ estimated Ibuprofen \\
\hline Potts and Guy & $\log \mathrm{kp}=0.71 \log \mathrm{P}-0.0081 \mathrm{MW}-2.74$ & 0.026 \\
Flynn and Amidon & $\log \mathrm{kp}=-1.44+0.79 \log \mathrm{P}-1.45 \log \mathrm{MW}$ & 0.022 \\
Wilschut et al. A & $\log \mathrm{kp}=-2.12+0.502 \log \mathrm{P}-\log \left(14.0+\mathrm{P}^{0.5}\right)$ & 0.009 \\
Wilschut et al. C & $\operatorname{Log~kp}=-1.55+0.481 \log \mathrm{P}-0.143(\mathrm{MW})^{0.5}$ & 0.020 \\
Wilschut et al. $\mathrm{D}$ & $\log \mathrm{kp}=-1.55+0.481 \log \mathrm{P}-0.143 \sqrt{\mathrm{MW}}$ & 0.020 \\
\hline
\end{tabular}

\section{CONCLUSIONS}

A monolithic hydrogel based topical patch was developed for skin delivery of IBU, based on a safe by design approach. An inverse correlation between PEG molecular weight and the release/permeation rate was observed pointing PEG 200 as the best enhancer. HPMC:Chitosan, in a ratio 1:5, and HPMC: Eudragit L30D-55 in a 1:1 proportion, $\%(\mathrm{w} / \mathrm{w})$, provided the best adhesive properties and promoted an improvement of drug in vitro release and permeation. Several mathematical models were used to elucidate the release mechanisms from the different patches and in vitro release kinetics showed to be driven by diffusion coupled with polymer relaxation mechanisms. The adhesive was considered the variable with more impact on permeation behaviour. A successful development of an innovative topical dosage form was carried out for IBU delivery, with HPMC: Eudragit L30D-55 (1:1) patch exhibiting a better performance than the commercial reference.

\section{DISCLOSURE STATEMENT}

The authors report no declarations of interest.

\section{ACKNOWLEDGEMENTS}

The authors acknowledge the Fundação para a Ciência e a Tecnologia (FCT), Portuguese Agency for Scientific Research, for financial support through the project PEst-UID/NEU/04539/2013, COMPETE (Ref. POCI-01-0145-FEDER-007440) and the Research Project n. ${ }^{\circ} 016648$ (Ref. PTDC/CTMNAN/2658/2014).

\section{REFERENCES}

Alexander A, Dwivedi S, Ajazuddin Giri TK, Saraf S, Tripathi DK. Approaches for breaking the barriers of drug permeation through transdermal drug delivery. J Control Release. 2012;164(1):26-40.

Aukunuru J, Bonepally C, Guduri V. Preparation, characterization and optimization of ibuprofen ointment intended for topical and systemic delivery. Trop J Pharm Res. 2007;6(4):855-860.

Bajaj $\mathrm{H}$ and Kumar T. Film forming gels: A review. Research Journal of Pharmaceutical, Biological and Chemical Sciences. 2016;7(4):2085-2091.

Benson HAE, Watkinson AC, Transdermal and topical drug delivery. John Wiley \& Sons, Hoboken, New Jersey. 2012.

Bolourchian N, Mahboobian MM, Dadashzadeh S. The effect of PEG molecular weights on dissolution behavior of simvastatin in solid dispersions. Iran J Pharm Res. 2013;12(Suppl):11-20.

Brown MB, Martin GP, Jones SA, Akomeah FK. Dermal and transdermal drug delivery systems: Current and future prospects. Drug Deliv. 2006;13(3):175-187. 
Bushra R, Aslam N. An overview of clinical pharmacology of ibuprofen. Oman Medical Journal. 2010;25(3):155-1661.

Can AS, Erdal MS, Gungor S, Ozsoy Y. Optimization and characterization of chitosan films for transdermal delivery of ondansetron. Molecules. 2013;18(5):5455-5471.

Caner C, Vergano PJ, Wiles JL. Chitosan film mechanical and permeation properties as affected by acid, plasticizer, and storage. J Food Sci. 1998;63(6):1049-1053.

Celebi D, Guy RH, Edler KJ, Scott JL. Ibuprofen delivery into and through the skin from novel oxidized cellulose-based gels and conventional topical formulations. Int J Pharmaceut. 2016,514(1):238-243.

Chang RK, Raw A, Lionberger R, Yu L. Generic development of topical dermatologic products: formulation development, process development, and testing of topical dermatologic products. AAPS J. 2013;15(1):41-52.

Cherukuri S, Batchu UR, Mandava K, Cherukuri V, Ganapuram KR. Formulation and evaluation of transdermal drug delivery of topiramate. Int J Pharm Inv. 2017;7(1):10-17.

Cilurzo F, Gennari CGM, Minghetti P. Adhesive properties: a critical issue in transdermal patch development. Expert Opin Drug Del. 2012;9(1):33-45.

Costa P, Sousa Lobo JM. Modeling and comparison of dissolution profiles. Eur J Pharm Sci. 2001;13(2):123-133.

Cox, Gad, Shayne, 2008. Pharmaceutical manufacturing handbook: production and Processes. Jonh Wiley \& Sons, pp. 267-311.

Dash M, Chiellini F, Ottenbrite RM, Chiellini E. Chitosan-A versatile semi-synthetic polymer in biomedical applications. Prog Polym Sci. 2011,36(8):981-1014.

Elias PM. Stratum corneum defensive functions: An integrated view. J Invest Dermatol. 2005;125(2):183-200.

Elias PM. The skin barrier as an innate immune element. Seminars in Immunopathology 2007;29(1):3-14.

Frederiksen K, Guy RH, Petersson K. The potential of polymeric film-forming systems as sustained delivery platforms for topical drugs. Expert Opin Drug Del. 2016;13(3):349-360.

Goldman RD. Efficacy and safety of acetaminophen versus ibuprofen for treating children's pain or fever: a meta-analysis. The journal of paediatrics 2005;146(1):142-143.
Safety Considerations for Product Design to Minimize Medication Errors. Guidance for Industry (04/06/2018)

https://www.fda.gov/regulatory-information/search-fdaguidance-documents/safety-considerations-product-designminimize-medication-errors-guidance-industry

Hadgraft J, Guy RH, Transdermal drug delivery systems: Second Edition, Revised And Expanded. Marcel Dekker, Inc, 2003. Chapter 1.

Herkenne C, Naik A. Ibuprofen Transport into and through the skin from topical formulations: in vitro- in vivo comparison. J Inv Dermatol 2007;127(1):135-142.

Heyneman CA, Lawless-Liday C, Wall GC. Oral versus topical NSAIDs in rheumatic diseases - A comparison. Drugs. 2000;60(3):555-574.

Idson B. Percutaneous absortion. J Pharm Sci. 1975;64(6): 901-924.

Iqbal N, Vitorino C, Taylor KMG. How can lipid nanocarriers improve transdermal delivery of olanzapine? Pharm Dev Technol. 2017;22(4):587-596.

Irfani G, Sunilraj R. Design and evaluation of transdermal drug delivery system of valsartan using glycerine as plasticizer. Int J Pharm Res. 2011;3:185-192.

Jorge LL, Carolina, CF, Teles VE. Topical preparations for pain relief: efficacy and patient adherence. J Pain Res. 2011;4:11-24.

Jung E, Lee EY, Choi HK, Ban SJ, Choi SH, Kim JS, Yoon IS, Kim DD. Development of drug-in-adhesive patch formulations for transdermal delivery of fluoxetine: In vitro and in vivo evaluations. Int J Pharm. 2015;487(1-2):49-55.

Kakhi, M., Prodduturi, S., Wokovich, A.M., Doub, W.H., Buhse, L.F., Sadrieh, N., Risk Analysis of Transdermal Drug Delivery Systems. Pharm Eng. 2007; 27(4).

Kathe K and Harsha K. Film forming systems for topical and transdermal drug delivery. Asian $\mathrm{J}$ Pharm Sci. 2017;12(6):487-497.

Luo L, Patel A, Sinko B, Bell M, Wibawa J, Hadgraft J, Lane ME. A comparative study of the in vitro permeation of ibuprofen in mammalian skin, the PAMPA model and silicone membrane. Int J Pharm. 2016;505(1-2):14-19.

Marks R. The stratum corneum barrier: The final frontier. J Nutr. 2004;134(8 Suppl):2017S-2021S. 
Menon GK. New insights into skin structure: scratching the surface. Adv Drug Deliver Rev. 2002;54(Suppl. 1):S3-S17.

Menon GK, Cleary GW, Lane ME. The structure and function of the stratum corneum. Int J Pharm. 2012;435(1):3-9.

Michels SL, Collins J, Reynolds MW, Abramsky S, ParedesDiaz A, McCarberg B. Over-the-counter ibuprofen and risk of gastrointestinal bleeding complications: a systematic literature review. Curr Med Res Opin. 2012;28(1):89-99.

Moser K, Kriwet K, Froehlich C, Kalia YN, Guy RH. Supersaturation: Enhancement of skin penetration and permeation of a lipophilic drug. Pharm Res. 2001;18(7): 1006-1011.

Nesseem DI, Eid SF, El-Houseny SS. Development of novel transdermal self-adhesive films for tenoxicam, an antiinflammatory drug. Life Sci. 2011;89(13-14):430-438.

Ong CKS, Seymour RA. An evidence-based update on nonsteroidal anti-inflammatory drugs. Clin Med Res. 2007;5(1):19-36.

Padula C, Colombo G, Nicoli S, Catellani PL, Massimo G, Santi P. Bioadhesive film for the transdermal delivery of lidocaine: in vitro and in vivo behavior. J Control Rel 2003;88(2):277-285.

Prausnitz MR, Langer R. Transdermal drug delivery. Nat Biotechnol. 2008;26(11):1261-1268.

Rainsford KD, 2015. Pharmacology and toxicology of ibuprofen, in: Rainsford, K.D. (Ed.), Ibuprofen: discovery, development and therapeutics. John Wiley \& Sons Ltd, Chichester, pp. 132-236.

Sarkar N. Thermal gelation properties of methyl and hydroxypropyl methylcellulose. J Appl Polym Sci. 1979;24(4):1073-1087.

Schmid-Wendtner MH, Korting HC. The $\mathrm{pH}$ of the skin surface and its impact on the barrier function. Skin Pharmacol Physi. 2006;19(6):296-302.

Silva CL, Pereira JC, Ramalho A, Pais A, Sousa JJS. Films based on chitosan polyelectrolyte complexes for skin drug delivery: Development and characterization. J Membrane Sci. 2008a;320(1-2):268-279.
Silva SMC, Pinto FV, Antunes FE, Miguel MG, Sousa JJS, Pais AACC. Aggregation and gelation in hydroxypropylmethyl cellulose aqueous solutions. J Colloid Interf Sci. 2008b;327(2):333-340.

Simoes, A, Veiga, F, Vitorino, C, Figueiras, A. A tutorial for developing a topical cream formulation based on the quality by design approach. J Pharm Sci. 2018;107(10):2653-2662.

Suto B, Berko S, Kozma G, Kukovecz A, Budai-Szucs M, Eros G, Kemeny L, Sztojkov-Ivanov A, Gaspar R, Csanyi E. Development of ibuprofen-loaded nanostructured lipid carrier-based gels: characterization and investigation of in vitro and in vivo penetration through the skin. Int J Nanomed. 2016;11:1201-1212.

Tombs EL, Nikolaou V, Nurumbetov G, Haddleton DM. Transdermal Delivery of Ibuprofen Utilizing a Novel SolventFree Pressure-sensitive Adhesive (PSA): TEPI ${ }^{\circledR}$ Technology. J Pharm Innov. 2018;13(1):48-57.

Vaile JH, Davis P. Topical NSAIDs for musculoskeletal conditions - A review of the literature. Drugs. 1998;56(5): 783-799.

Valenta C, Wanka M, Heidlas J. Evaluation of novel soyalecithin formulations for dermal use containing ketoprofen as a model drug. J Control Rel. 2000;63(1-2):165-173.

Vinod S, Sharma H. Topical non steroidal anti-inflammatory drug (NSAIDs) microemulsions: Rationale, review and future prospective. Asian J Pharm. 2014;7.

Vitorino C, Almeida J, et al. Co-encapsulating nanostructured lipid carriers for transdermal application: From experimental design to the molecular detail. J Control Rel. 2013;167(3): 301-314.

Willard-Mack C, Ramani T, Auletta C. Dermatotoxicology: safety evaluation of topical products in minipigs: study designs and practical considerations. Toxicol Pathol. 2016;44(3):382-390.

Received for publication on $06^{\text {th }}$ August 2018 Accepted for publication on $01^{\text {st }}$ January 2019 